\title{
AN INTRODUCTION OF MULTISLICE CT (MSCT) AND ONE YEAR OF EXPERIENCE IN BIRENDRA HOSPITAL
}

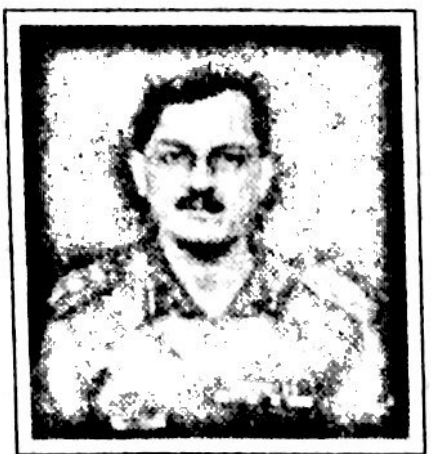

Since its clinical introduction in 1991, volumetric $\mathrm{CT}$ scanning using spiral or helical scanners has resulted in a revolution for diagnostic imaging. In addition to new application for CT such as CT angiography and assessment of patient with renal colic, many routine applications such as the detection of lung and liver lesions have substantially improved. Helical CT has improved over the past ten years with faster gantry rotation, more powerful X-ray tubes and improved interpolation algorithm (1). However in practice the spiral data sets from monoslice system suffered from a considerable mismatch between the transverse and longitudinal spatial resolution. The introduction of sub-second spiral scanning with Somatom Plus 4 at RSNA-1994 was a first step to facilitate routine clinical work with respect to scanable volume, total scan time and axial resolution.

The greatest advance has been the recent introduction of Multislice CT(MSCT) scanners. At RSNA 1998 this new technology has been introduced by several manufacturers representing an obvious

\author{
SCOL DR. SHIVA BASNET, MD
}

Consultant Radiologist

quantum leap in clinical performance (2-4). Currently capable of acquiring 4 channels of helical data simultaneously MSCT scaners have achieved the greatest incremental gain in scan speed and have profound implication for clinical CT scanning. Fundamental : advantage of MSCT include substantially 1 shorter acquisition time, retrospective : creation of thinner or thicker sections from s the same raw data and improved three $i$ dimentional rendering.

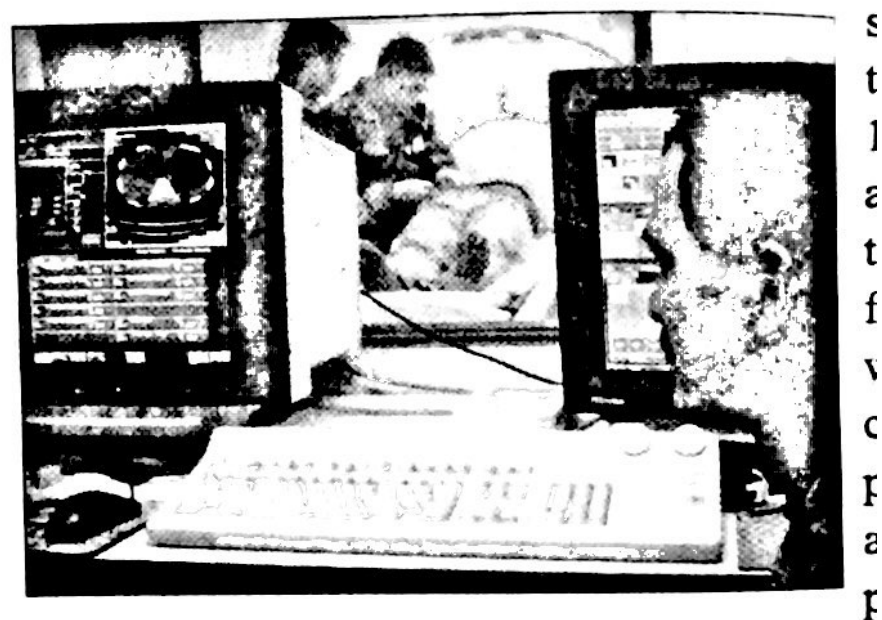

\section{Clinical applications}

The advantages of MSCT are important tc $s$ many applications of CT scanning including $\mathbf{\epsilon}$ oncologic or trauma patients anc $s$ characterization of the focal lung and live lesions.However greatest impact has been or 1 CT angiography, cardiac imaging, calciur I scoring, Virtual endoscopy, colonoscopy virtual bronchoscopy and high resolutior ${ }^{i}$ imaging (5). 


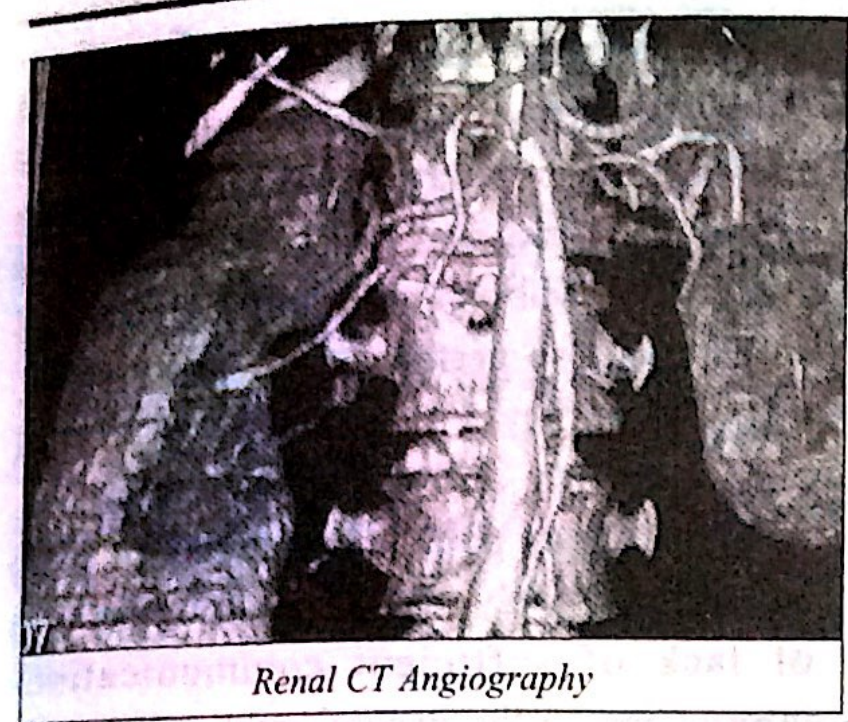

\section{CT angiography}

It has the ability to obtain a $1^{\text {st }}$ circulation study of a rapidly injected contrast bolus with thinner images. As a result of shorter acquisition time, contrast dose can be significantly reduced. CT angiography of intracranial vessels benefits from quick and detailed scanning technology of MSCT. At $1 \mathrm{~mm}$ collimation, circle of willis can be scanned within 10 seconds. This permits the entire scan to be completed during the $1^{\text {st }}$ pass of contrast material through the arterial system. For CT angiography of thoracoabdominal aorta volume coverage is from thoracic inlet to the inguinal region which can be covered in 20 seconds. A complete lower extremity study can be performed from renal vascular pedicles to the ankle in the same manner. Similarly pulmonary arteries, visceral branch vessels can be performed significantly with increase spatial resolution. The ability to cover the entire chest in 10 seconds allows the scanning of children without sedation.

\section{High resolution imaging}

Imaging of temporal bone is improved using MSCT because in-plane resolution can be increased. The delicate structures of middle and inner ear are sharply delineated and even subtle changes can be assessed.

\section{Multiplanner imaging capability}

Convetionally CT images are acquired and usually viewed in the transvers plane. With MSCT entire or part of the body is rapidly imaged with thin collimation $(1-3 \mathrm{~mm})$ during a single breath hold. This produces a data set that can be manipulated and reviewed on a work station to produce high quality multiplanner reformation (6). Transverse CT is useful and adequate for most indications, however there are clinical scenarios and disease states in which sagittal, coronal and curved multiplanner reformation can be more definitive or contributory to a diagnosis.

\section{Multiphagic imaging capability}

Hepatic circulation has two major components, arterial $25 \%$ and protal venous $75 \%$. A rapidly injected contrast bolus will opacify the liver in two stages, in an initial hepatic arteryphase followed by a portal venous phase. The appearance of focal hepatic mass on dynamic contrast enhanced CT depends upon the vascularity of tumour. Most benign or malignant tomour receive blood from arterial system, exceptions being regenerating liver nodules and well differentiated hepatocelluar carcinoma which have blood supply similar to hepatic parenchyma. With monosclice technology it is not possible to obtain a pure arterial phase because of the admixture of hepatic arterial and portal venous enhancement on the $1^{\text {st }}$ imaging pass. MSCT scanners however has the ability to scan through the entire liver in 10 seconds or less and allows acquisition of two separate sets of CT images of the liver within the time generally regarded as the hepatic arterial dominant phase (7). MSCT with its increased speed and resolution 
proves to be superior for detection and characterization of focal liver regions.

\section{One year of Multislice CT in Birendra Hospital}

Multislice CT was installed in our hospital on $14^{4}$ Poush 2059 B.S. Since that time we have been doing almost all possible imagings numbering around 1000 cases.

Distribution of CT imagings in the last year

\begin{tabular}{|c|c|c|c|c|c|c|c|c|}
\hline$\widehat{S \mathrm{Na}}$ & $\begin{array}{l}\text { Diwntucion of } \\
\text { the patients }\end{array}$ & Head & Chest & Neck & $\begin{array}{l}\text { Abdt+ } \\
\text { Pelvis }\end{array}$ & $\begin{array}{l}\text { USpine, } \\
\text { D/Spine }\end{array}$ & Other & Toul \\
\hline 1. & Requer Army & 176 & 13 & 10 & 11 & 19 & 6 & 235 \\
\hline 2 & Extrifuly & 345 & 79 & 37 & 33 & 130 & 20 & 644 \\
\hline 3 & Civil & 85 & 24 & 11 & 2 & 20 & 3 & 145 \\
\hline \multicolumn{2}{|c|}{ Grent Then } & 606 & 116 & 58 & 46 & 169 & 29 & 1024 \\
\hline
\end{tabular}

\section{Other procedures}

1. Renal CT angiography

8.

2. Abdominal \& thoracic aorta CT angiography

3. Circle of Willis angiography

4. Virtual clonoscopy

5. Virtual bronchoscopy

6. Myelo CT

7. CT guided biopsy Total

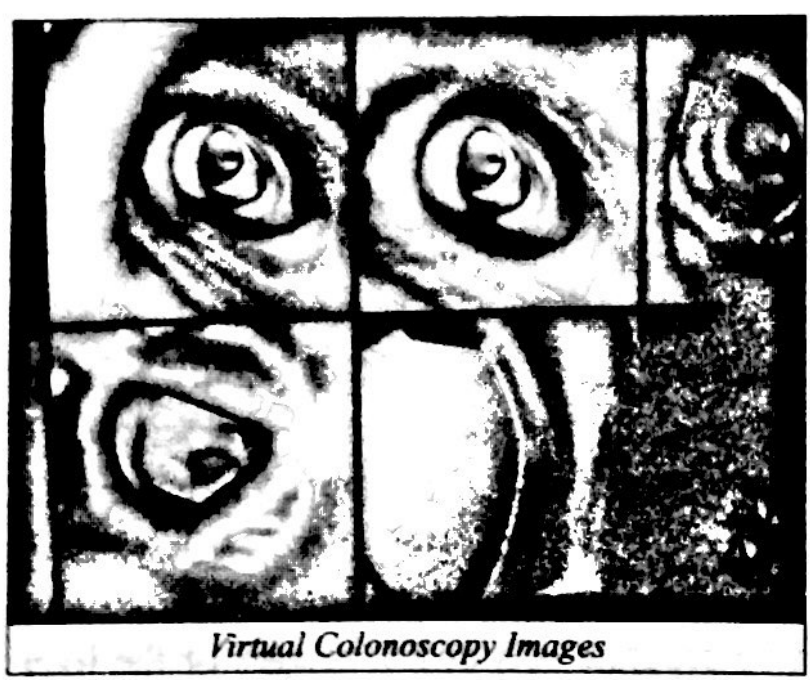

From the above table it is learn that maximum number of CT scanning was done among
Ex-serviceman, their families and familie of regular army followed by regular arm. and civilian population. Among the part examined, head scan was most frequentl. asked followed by spines, chest, abdomen anc so on. We also performed CT angiography CT myelography, Virtual clonoscopy, Virtua bronchoscopy and CT guided biopsies Among civilian population number of case are comparatively less. It could be becaust of lack of sufficient communication information to the general public. Howeve in near future we expect to increase the number of patients by increased awarenes? among the public about this facility in our hospital.

\section{References}

1. Kalender WA, Seissler W, Klotz E, Vock P. Spira volumatric $\mathrm{CT}$ with with single-breath-hold technique, continuous transport and continuous scanner rotation, Radiology 1990; 176: 181-83.

2. Schaller $\mathrm{S}$, Flohr $\mathrm{T}$, Wolf $\mathrm{K}$, Kalender WA Evaluation of the reconstruction algorithm for multirow -CT. Radiology 1999; 209 (P): 434.

3. Taguchi K, Aradate H. Algorithm for image reconstruction in multislice helical CT. Med Phys 1998; $25:$ 550-561.

4. Fox SH, Tanenbaum LN, Ackelsberg S, He HD, Hsieh J, Hu H. Future direction in CT technology, Neuroimaging Clinics of North America. 1998; 8: 497-513.

5. Berland LL, Smith JK. Multidetector-array CT: once again, technology creates new opportunities. Radiology 1998; 209: 327-21.

6. Wong K, Paulson EK, Nelson RC. Breath-hold three-dimensional CT of the liver with multi. detector row helical CT. Radiology 2001; 219: 75-79.

7. Murakami M, Kim T, Takahashi S, Hori M Takamura M, Nakamura H. Ekiration of optimal timing of the arterial phase imaging for the detection of hypervascular hepatocelluar carcinoma by using triple arterial phse imaging of Multidetector-row helical CT. Radiology 2000; 217(P):367

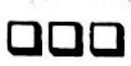

